\section{Avaliação da notificação de óbitos de menores de um ano ao Sistema de Informações da Atenção Básica (SIAB)}

\section{An evaluation of the registration of deaths of infants aged less than one year in the Basic Care Information System (SIAB)}

Paulo Germano de Frias 1

Milde do Rego Barros Alves Cavalcanti 2

Pricila Honorato Mullachery 3

Giseli Nogueira Damacena 4

Célia Landmann Szwarcwald 5

1 Instituto de Medicina Integral de Pernambuco Prof. Fernando Figueira. Rua dos Coelhos, 300. Boa Vista. Recife, PE, Brasil. CEP: 50.070-550. Email: pfrias@imip.org.br

2,3 Secretaria de Saúde do Recife. Recife, PE, Brasil

4,5 Laboratório de Informações em Saúde. Instituto de Comunicação, Informação Científica e Tecnológica em Saúde. Fundação Oswaldo Cruz. Rio de Janeiro, RJ, Brasil.

\begin{abstract}
Objectives: to assess the quality of information on deaths of infants aged less than one year registered in the Basic Care Information System (SIAB).

Methods: a quality assessment of SIAB data in the municipality of Recife. Changes in the SIAB data on mortality were compared over the period between 2000 and 2006, using municipal and federal databases available on the DATASUS site. For 2006, deaths of infants aged under one year registered in the municipal SIAB were given individual details and semi-structured interviews with community health officers (ACS) were used to confirm or reject registered deaths, by identifying errors a weaknesses in the production of SIAB data.

Results: there are differences between the federal and municipal SIAB databases. Only $34.0 \%$ of deaths registered by ACS were confirmed. The errors that led to over-reporting of deaths in the SIAB included errors relating to the definition of the event (4.6\%), the filling in of forms $(33.8 \%)$, consolidation $(7.3 \%)$ and typing of data $(21.4 \%)$.

Conclusions: although there is evidence of the potential of the SIAB to monitor and evaluate primary care, there are still weaknesses in the information production process and efforts need to be made to ensure more reliable data are collected.
\end{abstract}

Key words Evaluation, Death, Child, Information systems, Primary health care

\section{Resumo}

Objetivos: avaliar a qualidade da informação sobre óbitos de menores de um ano notificados ao Sistema de Informação da Atenção Básica (SIAB).

Métodos: estudo avaliativo da qualidade dos dados do SIAB realizado no município do Recife. Procedeu-se a análise do comportamento dos dados do SIAB, comparativamente aos do sistema de informação sobre mortalidade no período de 2000 a 2006, utilizando as bases municipal e federal disponível no site do DATASUS. Para 2006, os óbitos de menores de um ano notificados ao SIAB municipal foram individualizados e a partir de entrevistas semiestruturadas com os agentes comunitários de saúde (ACS) as notificações foram confirmadas ou descartadas, identificando erros, e fragilidades no processo de produção dos dados do SIAB.

Resultados: existem diferenças entre as bases de dados federal e municipal do SIAB. Apenas 34,0\% dos óbitos notificados pelos ACS, foram confirmados. Entre os erros que levaram a notificação excessiva de óbitos no SIAB destacam-se os relacionados à definição do evento (4,6\%), os de preenchimento $(33,8 \%)$, consolidação $(7,3 \%)$ e digitação $(21,4 \%)$ dos dados.

Conclusões: apesar da disponibilidade de indicadores e reconhecimento quanto às potencialidades do SIAB para o monitoramento e avaliação da atenção primária, este permanece com fragilidades no processo de produção da informação, necessitando de investimentos para garantir maior confiabilidade dos dados.

Palavras-chave Avaliação, Óbito, Criança, Sistemas de Informação, Atenção básica à saúde 


\section{Introdução}

O Sistema de Informação da Atenção Básica (SIAB) é uma das fontes de dados utilizadas pelo Ministério da Saúde (MS) como instrumento de monitoramento da Estratégia de Saúde da Família e do Programa de Agentes Comunitários de Saúde (PACS) no Brasil. ${ }^{1}$ O sistema foi criado em 1998 a partir do Sistema de Informações do Programa de Agentes Comunitários de Saúde (SIPACS), após a incorporação de outros profissionais de saúde às equipes de saúde da família (ESF), juntamente com o agente comunitário de saúde (ACS), nos cuidados de atenção primária à saúde da população.1,2

Quando comparado aos Sistemas Nacionais de Informação em Saúde como o Sistema de Informações sobre Mortalidade (SIM), Sistema de Informações sobre Nascidos Vivos (Sinasc), Sistema de Informações de Agravos de Notificação (Sinan), entre outros, de racionalidade epidemiológica, para captação e registro de eventos vitais ou para a vigilância de agravos à saúde, o SIAB, diferentemente, foi pensado sob a lógica gerencial, para o monitoramento e avaliação da atenção primária.1,3,4

O sistema incorporou conceitos específicos advindos da proposta da atenção primária do Sistema Único de Saúde (SUS), como territorialização, responsabilidade sanitária e a micro-espacialização de problemas de saúde 2 possibilitando o diagnóstico demográfico e sócio-sanitário da população, o acompanhamento de grupos prioritários e das famílias da área coberta pelas ESF e PACS além das atividades realizadas pelos profissionais. 1

A disponibilidade de dados referente ao número de óbitos de menores de um ano e o de nascidos vivos (NV) no SIAB favoreceu a proposição do seu uso como fonte complementar de informação para captação destes eventos vitais em municípios de pequeno porte e com precariedade dos dados do SIM e do Sinasc e elevada cobertura de atenção primária. ${ }^{5-7}$ Mello Jorge et al. ${ }^{8}$ recomendam sua utilização como fonte alternativa para a redução do sub-registro no SIM e Sinasc, pela proximidade dos ACS com a ocorrência dos eventos.

Entretanto, existem questionamentos sobre a contribuição do SIAB na recuperação das informações vitais em municípios de diferentes portes populacionais e com diferentes níveis de qualidade dos dados do SIM.

Este trabalho tem o objetivo de avaliar a qualidade da informação sobre os óbitos de menores de um ano notificados ao SIAB no ano de 2006, em um município de grande porte do nordeste brasileiro.

\section{Métodos}

Estudo avaliativo da qualidade dos dados sobre óbito de menores de um ano notificados ao SIAB no município do Recife, capital do Estado de Pernambuco, cuja seleção se deveu à elevada cobertura da atenção primária e pela alta qualidade das informações do SIM.

O município conta com uma população de 1.515.000 habitantes distribuídas em $209 \mathrm{Km}^{2}$ e possui uma rede de saúde para todos os níveis de complexidade. Em 2006, a rede de atenção primária era composta por 28 unidades básicas tradicionais e 110 unidades de saúde da família nas quais atuavam 217 ESF e 1804 ACS que, em conjunto, assistiam aproximadamente $78 \%$ da população. 9

A estratégia do estudo envolveu duas etapas:

a) primeira etapa, para descrever o comportamento dos dados sobre óbitos menores de um ano e NV registrados no SIM, Sinasc e SIAB no período de 2000 a 2006 e as variações existentes entre as bases de dados do SIAB federal e municipal.Foram utilizados dados brutos de óbitos de menores de um ano existentes no SIAB e no SIM, e os de NV no Sinasc. Os dados da base municipal foram cedidos pela Secretaria de Saúde do Recife9 e os da base federal que estão disponíveis no site do DATASUS/MS (www.datasus.gov.br). Para identificar variações das informações foi calculada a razão de nascidos vivos e óbitos de menores de um ano entre o SIAB e os do Sinasc e SIM. A seguir calculou-se o Coeficiente de Mortalidade Infantil (CMI) por esses sistemas;

b) segunda etapa, para identificar as diferenças existentes entre as bases municipais do SIAB e a do SIM e os problemas no processo de produção dos dados do SIAB. Realizou-se uma pesquisa de campo sobre os óbitos de menores de um ano registrados no SIAB municipal e em particular a sua identificação nominal, em virtude dos relatórios do sistema disponibilizarem apenas informações agregadas. A coleta de dados, através de entrevistas semiestruturadas com os ACS, ocorreu no período compreendido entre outubro de 2007 a janeiro de 2008.

Os dados do SIM foram tomados como padrão ouro porque o número de óbitos menores de um ano das bases federal e municipal foi igual e todos estes são validados e investigados pela equipe da Secretaria de Saúde do Recife. ${ }^{9}$ A investigação é realizada no domicílio e através da revisão de prontuários (ambulatorial e hospitalar), revisão dos endereços registrados no sistema e recuperação de variáveis sem informação na Declaração de Óbito (DO). 
A Figura 1 sintetiza o percurso metodológico adotado no estudo entre o registro do óbito de menor de um ano no SIAB e a sua confirmação ou não, sinalizando as situações e tipos de erros identifi- cados. A partir dos óbitos de menores de um ano registrados no SIAB, foram mapeadas as microáreas em que havia notificação e os respectivos ACS responsáveis.

\section{Figura 1}

Fluxo da pesquisa para confirmação dos óbitos registrados no Sistema de informação da Atenção Básica- SIAB.

Entrevista com ACS* da microárea que registrou o óbito e análise documental

Óbitos de $<1$ ano registrado no SIAB

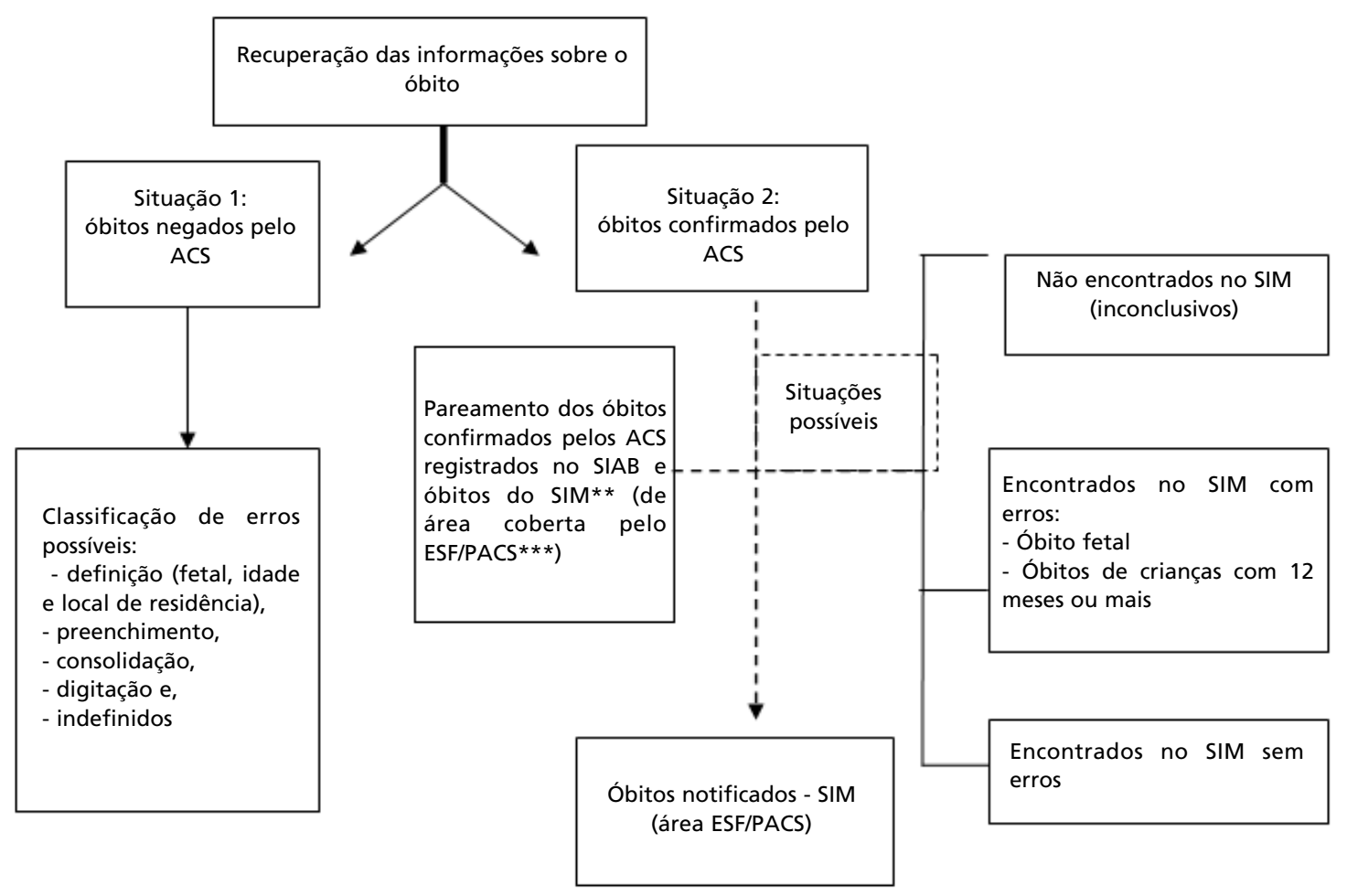

*Agente Comunitário de Saúde; ** Sistema de Informação sobre Mortalidade; ***Equipe de Saúde da Família/ Programa de Agentes Comunitários de Saúde. 
Os ACS que tinham óbitos registrados em suas microáreas foram entrevistados mediante questionário elaborado especificamente para este fim contendo questões referentes à identificação da mãe (nome e endereço) e ao óbito: data do óbito, causa do óbito registrada no SIAB, tipo do óbito e idade da criança. Também foram analisados os originais das fichas e relatórios do SIAB, prontuários e cópias das DO arquivadas nas Unidades de Saúde (US) de referência do ACS notificador do óbito.

A recuperação das informações sobre os óbitos registrados no SIAB, através das entrevistas e análise documental ensejou duas situações, a primeira em que os ACS negaram a existência do óbito notificado e a segunda em que os óbitos foram confirmados.

Quando o óbito não foi confirmado pelo ACS, classificaram-se os erros como: de definição, quanto ao tipo do óbito (fetal classificado como não fetal); a faixa etária (criança maior 12 meses) ou ao local de residência (residentes de outros municípios); de preenchimento da ficha do ACS (ficha D); de consolidação do relatório mensal (Relatório de Situação de Saúde e Acompanhamento - SSA2) ou erro de digitação. A ficha D é o instrumento de registro das atividades dos ACS e de eventos ocorridos durante o mês. O relatório SSA2 é o consolidado das fichas D de uma equipe de PACS ou PSF e que alimenta o SIAB. ${ }^{1}$ Os três primeiros erros, de definição, foram classificados com base na entrevista relatada pelo ACS. Os erros de preenchimento, consolidação e digitação foram verificados pelo entrevistador exclusivamente através da visualização das fichas ou relatórios. Quando as fichas não foram encontradas, o erro foi classificado como "erro de registro indefinido".

Quando o óbito foi confirmado pelo ACS, confrontaram-se as informações obtidas pelo pareamento desses óbitos e a base do SIM emergindo três situações: óbitos de menores de um ano encontrados no SIM sem erros; encontrados no SIM, porém com erros (óbitos fetais e de crianças com 12 meses ou mais) e os não encontrados no SIM caracterizados como inconclusivos.

Os resultados foram apresentados e analisados segundo o modelo de atenção ao qual o ACS era ligado, PACS ou ESF. Os erros foram classificados e identificados segundo os principais atores envolvidos.

Para a análise dos dados foi utilizado o programa estatístico SPSS versão 13.0.

O estudo foi aprovado pelo Comitê de Ética e Pesquisa em Seres Humanos, do Instituto de Medicina Integral Prof. Fernando Figueira (IMIP) com a carta de anuência do município.

\section{Resultados}

A Tabela 1 mostra que em 2006 o SIAB indicou a existência de 335 óbitos de menores de um ano notificados por 249 ACS, sendo 183 de ESF e 66 de equipes do PACS (dados não apresentados na tabela). No mesmo ano o SIM indicou a ocorrência de 327 óbitos dos quais 270 ocorreram em áreas cobertas pelas ESF e do PACS.

Dos 335 óbitos registrados no SIAB, 329 foram recuperados junto aos ACS durante a entrevista e seis óbitos foram considerados perdas, pois os ACS notificadores e/ou os enfermeiros supervisores haviam saído da equipe no período da pesquisa.

A razão de óbitos de menores de um ano entre os sistemas SIAB e o SIM variou bastante, sendo contrária ao aumento da cobertura da ESF/PACS nos dois primeiros anos, quando a cobertura aumentou e a razão diminuiu. Em 2003, a cobertura da ESF/PACS era de aproximadamente $50 \%$ e a razão de óbitos era de 1,0. Em 2006, o número de notificações de óbitos do SIAB ultrapassou o do SIM, na razão de 1,1 .

Em relação aos nascidos vivos, a razão $\mathrm{SIAB} /$ Sinasc não apresentou muitas variações. $\mathrm{Na}$ comparação entre o CMI calculado com os dados do SIAB e o CMI calculado com os do SIM/Sinasc, observa-se que a razão ultrapassou o valor 2,0 nos anos de 2003 e 2006, indicando que a mortalidade na área de cobertura do ESF/PACS teria sido duas vezes maior que o valor da mortalidade do município.

A diferença entre o número de $\mathrm{NV}$ e óbitos infantis registrados no SIAB municipal e na base federal chega a cerca de $1300 \mathrm{NV}$ e 55 óbitos a mais no SIAB municipal no ano de 2001 , enquanto que nos anos de 2000 e 2006 observou-se maior número de óbitos no SIAB nacional.

Dos 329 óbitos contidos no SIAB municipal com recuperação de informações, apenas $34 \%$ foram confirmadas pelos ACS como óbitos de menor de um ano ocorridos nas áreas de abrangência. Dentre estes, 11 foram excluídos após confronto com o SIM (dois registrados no SIM como óbito fetal e nove não encontrados no SIM/inconclusivos), resultando em apenas 101 óbitos. Foi observado maior percentual de óbitos verdadeiros entre os ACS das ESF (Tabela 2).

Os nove casos inconclusivos representam as situações em que: a) os óbitos não foram encontrados no SIM municipal, ou na ausência de evidências de se tratar de óbito de menor de um ano residente em 2006; b) quando a confirmação dos dados foi impos- 


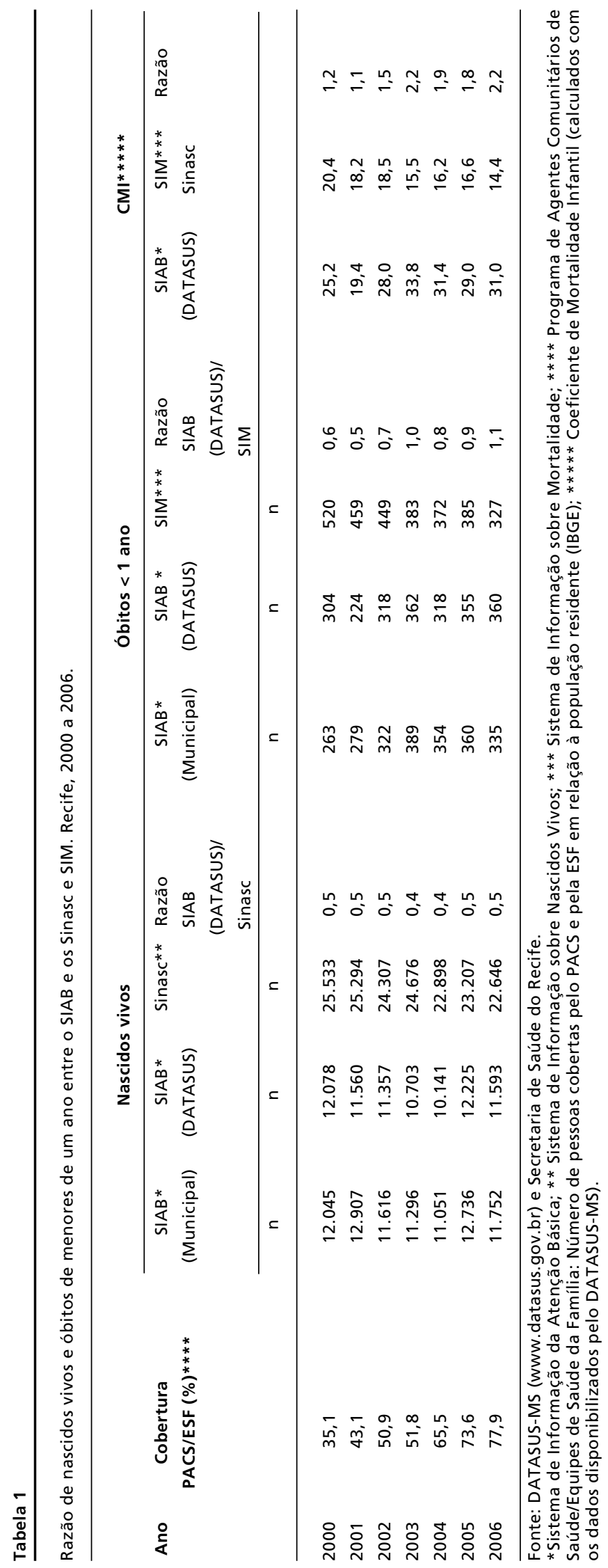


sibilitada pela saída definitiva do ACS da equipe ou pela mudança de endereço da família da criança.

Dentre os 219 erros de notificação, 217 foram identificados no momento da entrevista e mais dois foram encontrados quando da comparação com os dados do SIM. A Tabela 3 mostra que entre os erros de definição foram encontrados: dez óbitos fetais e um de criança com mais de 12 meses notificados como óbitos de menores de um ano; seis óbitos de não residentes ou ocorridos em outros anos.

Os erros de preenchimento representaram 33,8\% do total de erros. Em 29,7\% dos casos a classificação foi de "erro de registro indefinido", em virtude das fichas D ou SSA2 não terem sido encontradas no arquivo das US ocasionando a impossibilidade de identificar onde ocorreu o erro, se no preenchimento ou na digitação no sistema (Tabela 3).

Tabela 2

Distribuição dos óbitos confirmados durante a pesquisa de campo com os agentes comunitários de saúde. Recife, 2006.

\begin{tabular}{|c|c|c|c|c|c|c|}
\hline \multirow{2}{*}{ Situação } & \multicolumn{2}{|c|}{ ESF* } & \multicolumn{2}{|c|}{ PACS * * } & \multicolumn{2}{|c|}{ Total } \\
\hline & $\mathrm{n}$ & $\%$ & $\mathrm{n}$ & $\%$ & $\mathrm{n}$ & $\%$ \\
\hline Óbitos de menores de um ano confirmados na entrevista & 84 & 35,1 & 28 & 31,1 & 112 & 34,0 \\
\hline Confirmados no SIM*** & 77 & 32,2 & 24 & 26,7 & 101 & 30,7 \\
\hline Encontrados no SIM com erros**** & 1 & 0,4 & 1 & 1,1 & 2* & 0,6 \\
\hline Não encontrados no SIM/inconclusivos & 6 & 2,5 & 3 & 3,3 & 9 & 2,7 \\
\hline Óbitos de menores de um ano negados na entrevista & 155 & 64,9 & 62 & 68,9 & 217 & 66,0 \\
\hline Total & 239 & 100,0 & 90 & 100,0 & 329 & 100,0 \\
\hline
\end{tabular}

* Equipe de Saúde da Família; **Programa de Agentes Comunitários de Saúde; *** Sistema de Informação sobre Mortalidade; $* * *$ Dois óbitos fetais confirmados pelo SIM, validado pela investigação municipal do óbito. 


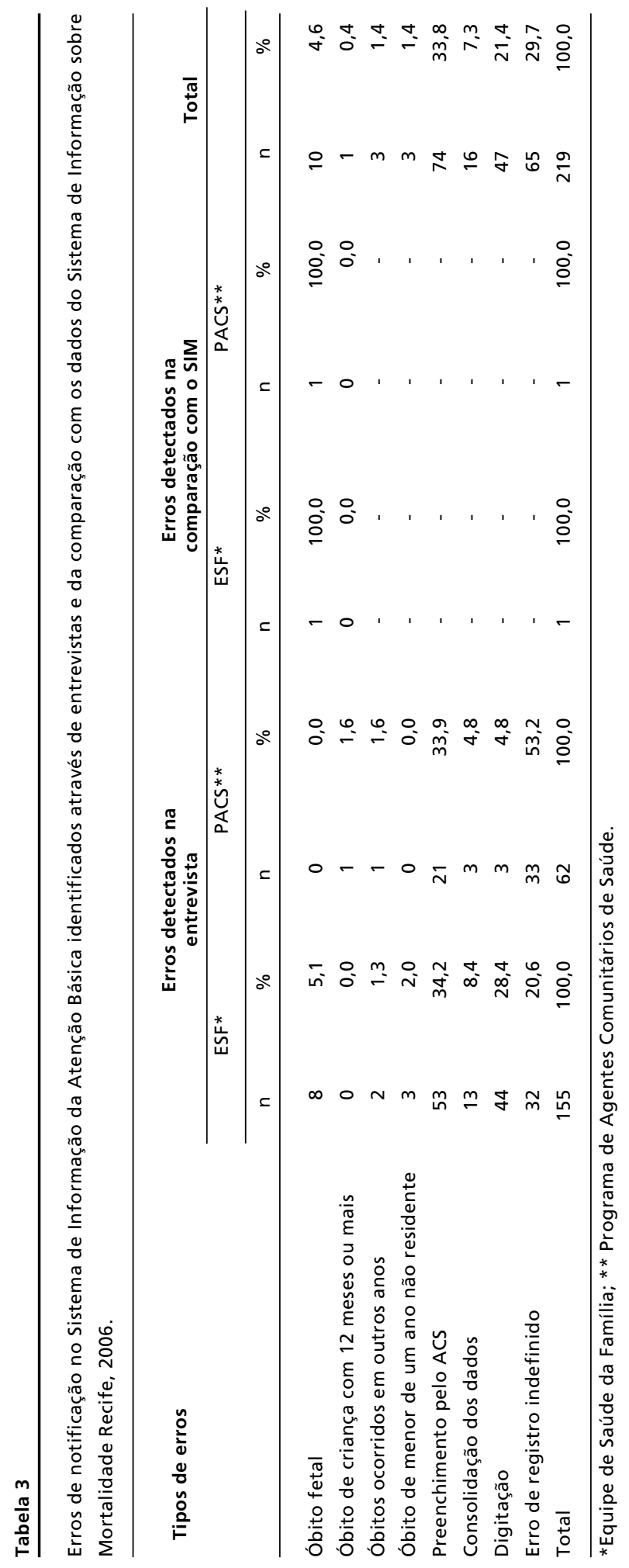




\section{Discussão}

No Brasil ao longo dos últimos anos vêm sendo realizados investimentos para ampliar a cobertura, a qualidade e o acesso às bases de dados dos sistemas de informação em saúde (SIS) com vistas ao monitoramento e avaliação da situação de saúde. $10 \mathrm{Com}$ frequência nas avaliações sobre os SIS se prioriza dimensões da qualidade relacionadas à cobertura, completude, clareza metodológica, confiabilidade, validade, entre outras. ${ }^{11}$

Apesar dos inquestionáveis avanços, os bancos de dados nacionais ainda apresentam limites e distorções, em particular as relacionadas ao subregistro de casos que comprometem o planejamento e o monitoramento de ações e intervenções públicas. 10

No padrão observado dos registros de NV e óbitos de menores de um ano no SIAB nas bases nacionais e municipais comparados aos do Sinasc e SIM para o período de 2000 a 2006 identificou-se diversos problemas operacionais. A despeito do aumento da cobertura do PACS e ESF no período, ${ }^{9} \mathrm{o}$ comportamento dos registros destes eventos vitais não se apresentaram coerentes.

Enquanto a razão dos nascidos vivos entre o SIAB e Sinasc se manteve praticamente inalterada, sugerindo subregistro do evento, a razão dos óbitos de menores de um ano entre o SIAB e o SIM apresentou oscilações no período analisado demonstrando a falta de regularidade nos registros dos eventos com implicações no uso dos dados como ferramenta de gestão para programação e planejamento de ações direcionadas a este público. Subregistro e falta de regularidade têm sido apontados como problemas frequentes em outros SIS. $6,12,13$

Por outro lado, as diferenças entre os dados do SIAB municipal e nacional explicitam problemas no fluxo das informações e transferência dos dados. A aparente perda de dados em alguns anos, assim como a duplicidade no nível nacional em outros anos, resulta em um padrão inconsistente, incompatível com o aumento da cobertura da estratégia. Esses diferentes comportamentos observados quanto ao registro dos eventos vitais no SIAB se constituem em dificuldades adicionais para o cálculo da taxa de mortalidade infantil para a área coberta pelo PACS e ESF. 4

Os resultados do presente estudo mostram que o registro de excesso de óbitos de menores de um ano, assim como o subregistro, $12-15$ também se constitui em um problema para a organização dos sistemas de informações. Apenas 34\% dos óbitos de menores de um ano originalmente notificados ao SIAB foram confirmados pelos ACS. Após confronto com os dados do SIM, esse precentual foi reduzido para $30 \%$ dos óbitos. Entretanto, o sobreregistro dos óbitos no SIAB não descarta a possibilidade de que existam óbitos de menores de um ano não notificados pelos ACS, situação não evidenciada em Recife, porém descrita em outros municípios. . $^{5}$

Diferentemente, um estudo que analisou as informações sobre hipertensos de três fontes, o Hiperdia, as fichas de aprazamento e o SIAB, mostrou que este último foi o que deteve maior ausência de registro sobre o agravo e que nenhuma das fontes apresentava registro universal dos casos. ${ }^{16}$ As diferentes direções observadas com respeito a notificação de eventos, o sobreregistro de óbitos de menores de um ano observado no presente estudo e o subregistro de hipertensos 16 apontam para a necessidade do uso de múltiplos métodos e técnicas na avaliação de SIS complexos e com grande quantidade de dados e eventos envolvidos.

Apesar do reconhecimento quanto as potencialidades do SIAB, 2,4,8,17,18 diversas críticas têm sido enumeradas, sendo as mais frequentes as relacionadas à cobertura incompleta, que só inclui a atenção primária realizada pelas ESF e PACS;18 a grande quantidade de fichas e relatórios;19 a ausência de informações relevantes, 19 como as sobre saúde bucal;20 a impossibilidade de identificação dos indivíduos no software; 19 a ausência da informação espacial compatível com a família/domicílio e a fragilidade das críticas na entrada de dados, entre outras.

Os problemas na produção da informação sobre óbitos de menores de um ano são diversos e têm sido descritas como recorrentes em outros estudos, $5,7,21$ destacando-se que se o software atualizasse automaticamente as idades a cada ano contribuiria para reduzir os erros. ${ }^{22}$

O excesso de formulários, ${ }^{19}$ a grande quantidade e diversidade de dados, o pouco espaço nos formulários, ${ }^{23}$ a insuficiente compreensão dos indicadores e das fichas do SIAB pela equipe, ${ }^{23-26} \mathrm{o}$ preenchimento das fichas como ato burocrático, a fragilidade na análise e monitoramento pelos níveis gerenciais e pelas equipes 19,24 e a baixa retroalimentação dos dados são alguns dos motivos citados como favorecedores da ocorrência de erros, que podem se repetir de forma sistemática ou aleatória durante o preenchimento, consolidação de uma ficha para a outra ou na digitação.

A insuficiência de capacitações sobre o SIAB tem contribuído para perpetuar os problemas. A realização de treinamentos de forma pontual, muitas 
vezes circunscritos apenas ao curso introdutório das ESF, é insuficiente para sanar as dificuldades das equipes para trabalhar com o sistema. ${ }^{19}$ No entanto, a capacitação permanente foi uma importante ferramenta para compreensão dos indicadores e preenchimento das fichas. ${ }^{24,26} \mathrm{O}$ manual do SIAB1 também é considerado insuficiente e superficial pelos profissionais das equipe. 19

Por outro lado, as insuficientes discussões sobre o processo de trabalho da equipe 27,28 e em particular sobre a produção do dado ${ }^{19}$ não têm favorecido a melhoria da informação. Uma síntese dos atores responsáveis pela produção das informações, sua participação nos erros encontrados e os principais fatores específicos e comuns relacionados aos erros podem ser observados na Tabela 4.

Outro aspecto que merece destaque são as implicações da inexistência de críticas automatizadas no SIAB, o que permite a manutenção de uma cadeia de erros, como a situação ilustrativa observada no estudo, em que foram notificados, em uma microárea, sete óbitos em um mês. $\mathrm{O}$ erro foi iniciado com o preenchimento incorreto, consoli- dado pelo supervisor e, finalmente, digitados no sistema, que aceita a entrada de um número indefinido de óbitos em uma mesma microárea.

O fluxo de informações da equipe para os outros níveis do sistema também agrega dificuldades. A partir de agosto de 2007, segundo Portaria $\mathrm{n}^{\circ} 1.876$, os dados do SIAB enviados mensalmente, seguindo a lógica do mês de competência, passaram a ser registrado do dia 21 do mês corrente até o dia 20 do mês subsequente. Essa mudança foi realizada com a justificativa de que se ao se concluir o mês no dia 20 , haveria tempo suficiente para os dados serem digitados e enviados as demais esferas gestoras antes do final do mês civil. Assim, os repasses de recursos do MS poderiam ser feitos mais rapidamente. Entretanto, essa diferença entre o dia de fechamento do SIAB e o último dia do mês se configura em uma dificuldade para a organização do fluxo de trabalho das equipes e a interpretação dos dados. Incoerências como esta, sugerem ser o município um mero prestador de serviços, à medida que só utilizam as informações para repasse as demais esferas. 29

Os achados deste estudo indicam a fragilidade

Tabela 4

Atores envolvidos e fatores relacionados aos erros identificados na pesquisa. Recife, 2006.

\begin{tabular}{|c|c|c|c|}
\hline \multirow{2}{*}{ Atores } & \multirow{2}{*}{$\begin{array}{l}\text { Erros na } \\
\text { produção dos } \\
\text { dados }\end{array}$} & \multicolumn{2}{|c|}{ Fatores relacionados ao erro } \\
\hline & & Fatores específicos & Fatores comuns \\
\hline & $\begin{array}{l}\text { Erro de definição do even- } \\
\text { to - óbito de menor de } \\
\text { um ano }\end{array}$ & $\begin{array}{l}\text { - Baixo conhecimento sobre } \\
\text { a terminologia empregada }\end{array}$ & $\begin{array}{l}\text {-Burocratização da produção } \\
\text { dos dados - simples preenchi- } \\
\text { mento das fichas. }\end{array}$ \\
\hline \multirow[t]{3}{*}{$\begin{array}{l}\text { ACS*/Enfermeiro } \\
\text { supervisor/supervi- } \\
\text { sor de área }\end{array}$} & $\begin{array}{l}\text { Erros de preenchimento/ } \\
\text { Consolidação }\end{array}$ & $\begin{array}{l}\text { - Modelo de ficha confuso, } \\
\text { com sobrecarga de dados }\end{array}$ & $\begin{array}{l}\text { - Fragilidade na análise e } \\
\text { retroalimentação dos dados } \\
\text { produzidos }\end{array}$ \\
\hline & & $\begin{array}{l}\text { - Abastecimento irregular } \\
\text { de fichas levando ao } \\
\text { reaproveitamento das } \\
\text { fichas e perda de dados }\end{array}$ & $\begin{array}{l}\text { - Fragilidade das ações de moni- } \\
\text { toramento dos indicadores. }\end{array}$ \\
\hline & $\begin{array}{l}\text { Erro de compreensão do } \\
\text { indicador }\end{array}$ & $\begin{array}{l}\text { - Existência de capacitações } \\
\text { apenas pontuais para o tra- } \\
\text { balho com o SIAB }\end{array}$ & $\begin{array}{l}\text { - Inadequações do sistema } \\
\text { informatizado e do conjunto de } \\
\text { indicadores utilizados }\end{array}$ \\
\hline \multirow{3}{*}{$\begin{array}{l}\text { Digitador/gerenci- } \\
\text { ador do Sistema de } \\
\text { Informação da } \\
\text { Atenção Básica }\end{array}$} & Erros de digitação & \multirow{2}{*}{\multicolumn{2}{|c|}{ - Modelo de ficha confuso, com sobrecarga de dados }} \\
\hline & & & \\
\hline & & \multicolumn{2}{|c|}{ - Fragilidade na análise dos dados produzidos } \\
\hline
\end{tabular}

*Agente Comunitário de Saúde. 
das ações de monitoramento e avaliação nas ESF e PACS no município. Essa fragilidade envolve, não somente, os níveis gerenciais do SIAB, como da própria atenção básica. Esse cenário pode ser ilustrado ao se observar a distribuição das portarias federais relativas à atenção primária entre 2003 e 2008 quando foram publicadas 608 , das quais apenas doze tratavam de sistemas de informação, monitoramento e avaliação. 30

Entre as limitações do estudo destaca-se o viés de memória considerando o tempo decorrido entre a ocorrência e a investigação. A natureza do evento pode ainda gerar a sua negação por parte da equipe pela íntima relação com a qualidade da assistência prestada.

A recuperação das fichas do SIAB nas US, imprescindível para identificação de alguns problemas operacionais do sistema nem sempre foi possível. Tal fato foi atribuído a não oferta de quantidade suficiente dos instrumentos de registro às equipes, determinando o seu reaproveitamento em

\section{Referências}

1. Brasil. Ministério da Saúde. Manual do Sistema de Informação de Atenção Básica. Brasília, DF; 1998.

2. Medina MG, Aquino R, Carvalho ALB. Avaliação da atenção básica: construindo novas ferramentas para o SUS. Divulg Saúde Debate. 2000; 21: 15-28.

3. Mota E, Carvalho DMT. Sistemas de Informação em Saúde. In: Rouquayrol MZ, Almeida Filho N. Epidemiologia e Saúde. Rio de Janeiro: MEDSI; 2003. p. 606 - 28.

4. Brasil. Ministério da Saúde. Monitoramento na atenção básica de saúde: roteiros para reflexão e ação. Brasília; 2004.

5. Frias PG, Vidal SR, Pereira PMH, Lira PIC, Vanderlei LC Avaliação da notificação de óbitos infantis ao Sistema de Informações sobre Mortalidade: um estudo de caso. Rev Bras Saúde Matern Infant. 2005; 5 (Supl. 1): 543-51.

6. Szwarcwald CL. Strategies for improving the monitoring of vital events in Brazil. Int J Epidemiol. 2008; 37: 738-44.

7. Frias PG, Pereira PMH, Andrade CLT, Szwarcwald CL. Sistema de Informações sobre Mortalidade: estudo de caso em municípios com precariedade dos dados. Cad Saúde Pública. 2008; 24: 2257-66.

8. Mello Jorge MHP, Gotlieb SLD. O Sistema de Informação de Atenção Básica como fonte de dados para os Sistemas de Informações sobre Mortalidade e sobre Nascidos Vivos. Inf Epidemiol SUS. 2000; 10: 7-18.

9. Recife. Plano Municipal de Saúde, 2006 - 2009. Recife saudável: inclusão social e qualidade no SUS. Recife, 2006. [acesso em 15 out 2006]. Disponível em: http://www.recife.pe.bov.br

10. Brasil. Ministério da Saúde, Organização Pan-Americana da Saúde, Fundação Oswaldo Cruz. A experiência brasileira em sistemas de informação em saúde. Produção e disseminação de informação sobre saúde no Brasil. v. 1. Brasília, meses subsequentes, à falta de local específico para arquivamento das fichas, favorecendo seu extravio, e à alta rotatividade dos enfermeiros supervisores do PACS e ESF.

Os resultados empíricos da investigação possibilitaram uma melhor compreensão dos problemas que envolvem a geração da informação na atenção primária e ao elencar os múltiplos pontos de estrangulamento e principais atores envolvidos na sua produção apontam oportunidades para sua superação.

A existência de um sistema de informação confiável e que possa subsidiar a tomada de decisão em saúde nos níveis locais é fundamental para a gestão da saúde e em particular das ESF.17,20 O SIAB tem potencialidades para servir como uma ferramenta de monitoramento e avaliação da atenção primária, 4,18 em que pese permanecer carecendo de ajustes quanto a sua concepção, face às novas necessidades de saúde, mas, sobretudo, quanto ao seu modo de operar.
DF: Ministério da Saúde; 2009.

11. Lima CRA, Schramm JMA, Coeli CM, Silva MEM. Revisão das dimensões de qualidade dos dados e métodos aplicados na avaliação dos sistemas de informação em saúde. Cad Saúde Pública. 2009; 25: 2095-109.

12. Szwarcwald CL, Leal MC, Andrade CLT, Souza-Júnior PRB. Estimação da mortalidade infantil no Brasil: o que dizem as informações sobre óbitos e nascimentos do Ministério da Saúde? Cad Saúde Pública. 2002; 18: 172536.

13. Almeida MF, Alencar GP, Schoeps D. Sistema de Informações Sobre Nascidos Vivos - Sinasc. Uma Avaliação de Sua Trajetória. In: Brasil. Ministério da Saúde. A experiência brasileira em sistemas de informação em saúde. Organização Pan-Americana da Saúde, Fundação Oswaldo Cruz. Brasília: Ministério da Saúde; 2009. 2 v(Série B. Textos Básicos de Saúde). p. 11-37.

14. Paes N. Avaliação da cobertura dos registros de óbitos dos estados brasileiros em 2000. Rev Saúde Pública. 2005; 39 : 882-90.

15. Frias PG, Pereira PMH, Andrade CLT, Lira PIC, Szwarcwald CL. Avaliação da adequação das informações de mortalidade e nascidos vivos no Estado de Pernambuco, Brasil. Cad Saúde Pública. 2010; 26: 671-81.

16. Girotto E, Andrade SM, Cabrera MAS. Análise de três fontes de informação da atenção básica para o monitoramento da hipertensão arterial. Epidemiol Serv Saúde. 2010; 19: $133-41$.

17. Samico I, Hartz ZMA, Felisberto E, Frias PG. A sala de situações na Unidade de Saúde da Família: o Sistema de Informação de Atenção Básica (SIAB) como instrumento para o planejamento estratégico local. Saúde Debate. 2002; 26: $236-44$. 
18. Medina MG, Aquino R, Carvalho ALB. Avaliando o programa de saúde da família. In: Souza MF, org. Os Sinais vermelhos do ESF. São Paulo: Hucutec; 2002. p. 135-44.

19. Silva AS, Laprega MR. Avaliação crítica do Sistema de Informação da Atenção Básica (SIAB) e sua implantação na região de Ribeirão Preto, São Paulo, Brasil. Cad Saúde Pública. 2005; 21: 1821-8.

20. Bittar TO, Meneghim MC, Mialhe FL, Pereira AC, Fornazari DH. O Sistema de Informação da Atenção Básica como ferramenta da gestão em saúde. RFO UPF. 2009; 14: $77-81$

21. Almeida MF, Alencar GP, Novaes HMD, Ortiz LP. Sistemas de informação e mortalidade perinatal: conceitos e condições de uso em estudos epidemiológicos. Rev Bras Epidemiol. 2006; 9: 56-68

22. Barbosa DCM. Sistemas de Informação em Saúde: a percepção e a avaliação dos profissionais diretamente envolvidos na atenção básica de Ribeirão Preto-SP [dissertação]. Ribeirão Preto: Faculdade de Medicina de Ribeirão Preto da Universidade de São Paulo; 2006.

23. Mota FRL. Registro de informação no sistema de informação em saúde: um estudo sobre a padronização nas bases SINASC, SIAB e SIM, no estado de Alagoas. 2009 [tese]. Minas Gerais: Universidade Federal de Minas Gerais; 2009.

24. Freitas FP, Pinto IC. Percepção da equipe de saúde da família sobre a utilização do Sistema de Informação da

Recebido em 2 de setembro de 2010

Versão final apresentada em 12 de dezembro de 2011

Aprovado em 12 de janeiro de 2012
Atenção Básica- SIAB. Rev Latino-am Enferm. 2005; 13: 547-54.

25. Marcolino JS. Informações em saúde: o uso do SIAB pelos profissionais das equipes de saúde da família [dissertação] Maringá: Universidade Estadual de Maringá; 2008.

26. Ferreira MEV, Schimith MD, Caceres NC. Necessidade de capacitação e aperfeiçoamento de profissionais de equipes de saúde da família da $4^{\mathrm{a}}$. Coordenadoria Regional de Saúde do Estado do Rio Grande do Sul. Ciênc Saúde Coletiva. 2010; 15: 2611-20.

27. Gribel EB. Atenção básica: do processo de trabalho ao sistema de informação [dissertação]. Rio de Janeiro: Escola Nacional de Saúde Pública Sérgio Arouca da Fundação Oswaldo Cruz; 2005.

28. Ribeiro EM, Pires D, Blank VLG. A teorização sobre o processo de trabalho em saúde como instrumental para análise do trabalho do Programa de Saúde da Família. Cad Saúde Pública. 2004; 20: 434-46.

29. Mishima SM, Villa TCS, Gomes ELR, Pratali MTR, Silva EM, Anselmi ML. O sistema de informação no processo gerencial dos serviços de saúde: algumas reflexões. Rev Latino-Am Enferm. 1996; 4: 83-9.

30. Castro ALB, Machado CV. A política de atenção primária no Brasil: notas sobre a regulação e financiamento. Cad Saúde Pública. 2010; 26: 693-705. 